\title{
Safety and risk in practice
}

Edited by André F. Tempelaar

Het Eiland 7, 7271 BK Borculo, The Netherlands

\section{Adverse patient occurrences in hospitals and the need for a conceptual approach}

After the Californian Medical Insurance Feasibility Study (CMIFS) in 1978 and the Harvard Medical Practice Study (HMPS) in 1990, another mega-study on adverse patient outcome in hospitals has been published in Australia [1]. The Quality in Australian Health Care Study (QAHCS) includes reviews of the medical records of a sample of 14,179 patients admitted to 28 hospitals in New South Wales and South Australia. The QAHCS was methodologically modelled on the HMPS; similar definitions were used and the same two-step review method by registered nurses and medical officers was followed. The outcome of the QAHCS was, however, quite different from that of the American studies. In the CMIFS, adverse occurrences were reported in $4.65 \%$ of all the patients admitted [2]. The HMPS, performed in the State of New York, reported that 3.7\% of the admitted patients experienced an adverse event during hospital stay [3]. However, in the Australian study a high proportion (16.6\%) of the medical records reviewed were associated with an adverse patient event. Although nearly 50\% of these adverse events occurred before admission, the rate of iatrogenic occurrences in hospitals was found to be more than twice as high as was reported in the HMPS and the CMIFS.

Nearly $14 \%$ of the patients in the Australian hospitals who experienced an adverse event remained permanently disabled and $5 \%$ of them died $(=0.8 \%$ of all admitted patients). The proportion of admissions associated with permanent disability or death increased with age; temporary disability was not associated with age. Adverse events accounted for seven additional days of stay in the hospital. A team of reviewers considered $51 \%$ of the adverse events preventable. Patients with diseases of the digestive tract or musculoskeletal and circulatory disorders were more at risk for adverse outcomes [1].

The alarming outcome of the QAHCS raises questions. Are Australian hospitals less safe than American hospitals? Are Australian patients more at risk? Or are the differences in outcome explained by a different interpretation of the analyzed data? John McNeil and Stephen Leeder suggest in the Medical Journal of Australia that the divergent outcomes are to be explained by methodological differences [4]. The interpretation of what constitutes an adverse event probably differed in the Boston review group and the Australian reviewers. The Australians may have used a wider explanation of what constitutes an adverse event than did their colleagues from Harvard. The Australian study group used the quality criterion "preventability", where the Boston group used the malpractice term "negligence". McNeil and Leeder also suggest that the documentation of patient records can play a role. More detailed documentation gives a better possibility for assessment of diagnostic and treatment procedures. Badly documented records can easily hide errors. The questions that remain are therefore:

- How accurate are estimations in adverse outcome studies? 
- What does this mean as regards the quality of hospital care?

- How can hospital care in different countries validly be compared?

- Which measures are required to remedy the problems identified?

The real incentive for the HMPS was concern about increasing malpractice claims and their economic consequences. The investigators concluded that there was little conformity between medical complications and malpractice litigation. Troyen Brennan from the Harvard School of Public Health (Boston, USA) reported that in New York hospitals less than $2 \%$ of negligence injuries were followed by a claim. No negligence could be proved in $80 \%$ of claims submitted [5]. In Australia quality incentives (to gain more insight in the problem) were the main reason for the study.

Brennan also suggests a difference in social medical circumstances between Australian and New York hospitals. Preliminary results (unpublished) of a study in hospitals in Utah and Colorado (USA), where the social conditions are more comparable to those in Australia, also give a lower figure for the adverse occurrence rate. Brennan proposes to test the methodological hypothesis by arranging a review of Australian records by American reviewers and a review of American records by Australians.

Retrospective judgement of negative outcomes must be interpreted carefully. Negative or adverse outcomes can all too easily be attributed to substandard care. Mistakes are apparently easy to spot in retrospect, but extremely difficult to detect at the time [6]. The results of the QAHCS may have been influenced by so-called hindsight bias.

These discussions of the studies performed in two countries illustrate some conceptual problems in the study of errors and accidents in health care. An error is part of the process but an accident is an outcome parameter. In many published studies process and outcome are confused. The lack of uniform definitions of errors, adverse occurrences and iatrogenic damage is another problem. Apples are easily taken for pears. There is a need for a conceptual approach to the problem.

A last conclusion may be that health care demands structural implementation of risk management systems in hospitals, and an alteration of attitude to arrive at a blame-free monitoring system.

\section{References}

[1] R.McL. Wilson, W.B. Runciman, R.W. Gibberd et al., The quality in Australian health care study, Med. J. Austr. 163 (1995), 458-471.

[2] D.H. Mills, Medical insurance feasibility study, West J. Med. 128 (1978), 360-365.

[3] President and Fellows of Harvard College, Patients, doctors, and lawyers: medical injury, malpractice litigation and patient compensation in New York, The Report of the Harvard Medical Practice Study to the State of New York, Boston, 1990.

[4] J.J. McNeil and S.R. Leeder, How safe are Australian hospitals?, Med. J. Austr. 163 (1995), 472-475.

[5] T.A. Brennan, Medical injuries: international perspectives, Med. J. Austr. 163 (1995), 475-476.

[6] J. Reason, Safety in the operating theatre. Part 2: Human error and organisational failure, Curr. Anaesth. Crit. Care 6 (1995), 121-126.

\section{The emotional impact of making mistakes: doctors need help, but fail to support their colleagues}

All doctors make mistakes, but not all doctors admit them. In the medical profession, imperfection and the notion of fallibility are still not accepted socially. "In a profession that values perfection, error is virtually forbidden", commented Marc Newman in his study about the emotional impact of mistakes made by family physicians [1]. 
John Ely (University of Iowa Hospitals and Clinics, Iowa City, USA) interviewed a random sample of 70 family physicians and general practitioners about errors they made and the perceived causes [2]. Ten physicians refused to participate and seven doctors could not remember making important errors! The remaining 53 physicians could remember over their professional lifetimes a mean number of errors of 10.7. The mean number of errors resulting in death was 1.2. Most of the reported errors concerned missed or delayed diagnoses. Most of these errors had an adverse outcome with tragical impact. The perceived causes, Ely found, were most commonly haste, distraction (emergency cases!), hesitation in making decisions, lack of knowledge, premature closure of the diagnostic process and inadequately aggressive patient management. Ely reported that all physicians had emotional problems about their role in making a mistake. Thirty percent behaved more defensively afterwards, e.g., adopting more aggressive diagnostic management.

In 1984 in the New England Journal of Medicine general practitioner David Hilfiker expressed in an emphatic way his feelings and emotions about his personal experience with mistakes [3]. He stated that physicians are often unable to face the mistakes they make. They feel isolated within their profession, with a silence around. As I reported before in this journal, Marc Conradi has described similar experiences among a group of Dutch general practitioners [4].

Marc Newman (Pennsylvania and Hahnemann University, Philadelphia, USA) interviewed 30 family physicians about serious errors and mistakes they had experienced [1]. Two physicians denied having made serious mistakes and five others were unsure whether they had committed an error. The others expressed strong emotional feelings about the incidents, ranging from self doubt, disappointment, self blame and shame to fear. All but one indicated strongly the need for support: talking to someone about the mistake, validation of the decision-making process, reaffirmation of professional competence and reassurance of personal self worth. Although they needed especially to talk about their problems with a colleague, they received support only from others; the greatest support came from their spouses. Some doctors experienced disinterest of colleagues, avoiding the dialogue, feeling uncomfortable about sharing the problem. One participant remarked: "So I closed off the issue and just tacked it away. Ultimately medicine tends to drive us away from our feelings and from getting close to the people. That behaviour makes it hard for us doctors to come together".

The family physicians were also confronted by Newman with a hypothetical case scenario, in which a colleague physician missed an obvious and fatal myocardial infarction. Nearly all the physicians recognized that their colleague was probably experiencing negative emotional feelings similar to their own. However, only nine of them were willing unconditionally to offer support. Most of them were unable to put aside feelings of shame and humiliation connected with the disclosure of mistakes.

Errors can be an opportunity to share pain with colleagues and receive their support. Medicine still attempts to deny the existence of errors and mistakes, however. It blames and sanctions the doctors working at the sharp end, rather than the medical system. Medicine simply has no place for mistakes, but we have to get more knowledge about the way physicians can prevent errors. "We need to know why good doctors sometimes make bad mistakes... We owe it to our patients and to ourselves to increase our knowledge about mistakes" [5].

\section{References}

[1] M.C. Newman, The emotional impact of mistakes on family physicians, Arch. Fam. Med. 5 (1996), 71-75.

[2] J.W. Ely, Perceived causes of family physicians' errors, J. Fam. Pract. 40 (1995), 337-344.

[3] D. Hilfiker, Facing our mistakes, N. Eng. J. Med. 310 (1984), 118-122. 
[4] A.F. Tempelaar, Preventing malpractice in primary care; the first goal is learning GP's to deal with errors, in: Safety and risk in practice, Int. J. Risk \& Safety Med. 8 (1996), 181-183.

[5] J.W. Ely, Physicicans' mistakes; will your colleagues offer support?, Arch. Fam. Med. 5 (1996), 76-77.

\section{Impaired to help and impaired to demand for help: doctors have problems with taking sick leave}

In the United Kingdom nearly one in three physicians is dissatisfied with his or her job. Particularly among general practitioners high levels of stress were reported, related with the way they perform their job, and long term stress is a major cause of illness. Several studies showed that health care providers are more at risk of psychosomatic illness and emotional stress. Depression, anxiety, burn out, alcohol and drug abuse were reported more among health care workers than in other comparable social groups [1]. Caplan reported symptoms of serious distress in $47 \%$ of 389 interviewed hospital consultants, general practitioners and senior health service managers in North Lincolnshire (UK) [2]. He found indications for clinical depression in $27 \%$ of general practitioners and $13 \%$ of them showed suicidal thinking.

However, when they become sick, doctors have a problem in demanding help and stopping their work. Comparing with other professionals, physicians make less use of health provisions and take sick leave less often when they are ill [3]. Cultural barriers (doctors' difficulty in seeking help) and organisational problems ('the doctor is indispensable') are the main causes of this behaviour.

NHS executive Gabriel Scally is worried about the inadequate support which Europe's largest employer, the UK National Health Service, gives to employed physicians when they become ill [3]. Other countries do better for their physicians, state GPs Ruth Chambers and Richard Maxwell [4]. In Canada, Norway and The Netherlands networks of confidential help services already exist. It has become clear that working in health care puts the workers more at risk for mental health problems, yet adequate help is not available. In daily practice the sick doctors become isolated. Mostly they treat themselves or consult a friendly doctor outside office hours, without establishing a normal doctorpatient relationship.

The working conditions of doctors must improve. Better services for sick doctors must be available and in all regions senior doctors must be appointed to act as confidence physicians, providing a help desk for doctors seeking assistance [4].

The basic problem leads back to medical education. The pathological way in which doctors think about and manage their own illness is developed in medical schools. They have learned to regard being sick as a sign of weakness. Doctors have become stigmatized by illness. They have learned to minimize their own emotional problems. They did not receive an opportunity to gain insight into their own illness problems. On the contrary, they learned to 'pull up their socks' when they were tired of 84 hours working with deprivation of sleep, or when they had a 'flu' and no one was there to replace them, or when they had emotional problems with the death of young patients or unsuccessful treatment, or with errors they should not have made. Medical education never taught them to avoid burning themselves out. Chambers and Maxwell call attention to the need for adequate help for sick doctors. They ask also for more fundamental research about ill-making factors in the health care services and for better management to prevent these.

Scally has raised another interesting question about the doctors illness: "Should patients have the right to know that their doctors have health problems that can affect their performance?" Patients have the right to get information about substantial risks of treatment. Unfortunately, the problem is that ill 
doctors often think that they will not do any harm to their patients. And, as part of the problem, their colleagues share this opinion. Ill doctors are often protected by their colleagues in an ostrich-like behaviour. In their study about the characteristics of physicians with alcohol problems Bissel and Jones reported this as much as twenty years ago [5]. Sick doctors have the right to be treated as sick patients.

\section{References}

[1] J. Firth-Cozens, Stress, psychological problems, and clinical performance, in: Medical Accidents, C. Vincent, ed., Oxford University Press, Oxford, 1993.

[2] R.P. Caplan, Stress, anxiety, and depression in hospital consultants, general practitioners, and senior health service managers, Br. Med. J., 309 (1994), 1261-1263.

[3] G. Scally, Physicians can't heal themselves, Lancet 347 (1996), 1059.

[4] R. Chambers and R. Maxwell, Helping sick doctors. Fix the job not the doctors, Br. Med. J. 312 (1996), $722-723$.

[5] L. Bissell and R.W. Jones, The alcoholic physician: a survey, Am. J. Psychiatry 133 (1976), 1142-1146.

\section{Surgeons infected with $\mathrm{HBV}$ or $\mathrm{HCV}$}

Surgeons are at risk for acquiring transmitted hepatitis infection. In the USA an estimated 1900 surgeons $(1.4 \%)$ are chronically infected with hepatitis B virus (HBV). Most commonly they have been infected by selfwounding during invasive procedures. Transmission of HBV from health care providers to patients is, however, uncommon; worldwide some 30 clusters of HBV transmission by surgeons have been reported, involving about 350 patients.

Health care providers clearly have to remain alert regarding this iatrogenic transmission of viral pathogens. In 1992 a 47-year old woman became ill with an acute hepatitis B after undergoing a thymectomy in an American hospital [1]. She was operated by an HBV-infected thoracic-surgery resident. The surgeon had experienced an acute HBV-infection six months earlier. Over a period of 12 months he had been involved in two hospitals in operations on 239 thoracic surgery patients. A retrospective re-examination was performed with 144 of these patients. Nineteen of them (13\%) had evidence of recent HBV infection. In an additional epidemiological study 124 patients operated by other thoracic surgeons were also re-examined. None of them had evidence of recent HBV infection. Causes other than transmission by the infected surgeon could not be found. The surgeon had respected hygienic and infection-preventing procedures and he was attested as having a good operating technique. Needle punctures were recalled only twice during the whole period. Blood samples from patients and doctor nevertheless confirmed the suspicion: the HBsAg subtype and the partial HBV DNA sequences from the surgeon were identical to those from 13 of the infected patients.

A possible explanation has been found in the procedure of suture tying. The surgeon complained about pain in his index fingers during prolonged suture tying. Other surgeons also reported this phenomenon. In a simulation performance of suture tying paper-cut-like lesions on the fingers were found. In the rinsing water HBsAg and HBV DNA were isolated. The combination of finger lesions and glove failure was possibly the cause of this epidemic of HBV infection. The tragic outcome for patients and surgeon could have been prevented if the surgeon had received HBV-vaccination. Although the surgeon was offered an HBV-vaccine, he never received it.

A similar story was reported by Juan Esteban et al. in Barcelona (Spain) relating to hepatitis C infection [2]. As a part of the Barcelona Post-Transfusion Hepatitis Study (BPTHS) two patients were 
found with acquired hepatitis $\mathrm{C}(\mathrm{HCV})$ after cardiac surgery with transmissions that could not be caused by blood transfusions. The surgeon who performed the invasive procedure was known to have chronic hepatitis C. All former patients of this surgeon were identified. Between 1988 and 1994 he had performed (or assisted with) surgery in 643 patients of whom 222 participated in follow-up studies of the BPTHS. Nineteen of 222 patients (8.6\%) had developed an acute hepatitis C postoperative; a transfusion related hepatitis $C$ was probably the cause in 13 of them. In the blood of five of the six remaining patients and in the blood of the surgeon the same genotype was found. All patients had undergone valve replacement. The results of a phylogenetic-tree analysis indicated nosocomial transmission of $\mathrm{HCV}$ from the surgeon to the patients.

This surgeon, again, had followed a proper procedure and could not be suspected of negligence. In 1984 he had injured himself with a scalpel while operating a patient with a chronic hepatitis B. He was treated with hepatitis B immunoglobulin and HBV-vaccine. Despite treatment he had developed a non-A-non-B hepatitis after six months. Since 1992 he had been treated with interferon alfa $2 \mathrm{~b}$. Specific questioning showed that the surgeon had often suffered small percutaneous injuries of his fingers. Most of these small injuries occurred in the course of tying the sutures during the closure of the sternum. In many cases he did not notice the injuries and perforation of the gloves until after the procedure. Other surgeons in the same hospitals also reported percutaneous injuries during the closure of the sternum with wire sutures.

Surgeons have a considerable professional risk of becoming infected with HBV and HCV during invasive procedures. Although uncommonly, they can transmit the infection to their patients. Julie Gerbering (University of California, San Francisco, USA) has wondered if guidelines for managing infections in health care providers should be readjusted [3]. Ironically most interest in prevention concerns HIV transmission, although only one cluster of HIV transmission from a health care provider to a patient has been reported until now (in a dental practice in Florida). Regular blood tests of health care providers performing invasive procedures can be a help for early detection of infected health care providers. Exclusion from invasive procedures can be considered.

The risk of adopting strict guidelines, however, may be a defensive attitude, i.e., refusal of surgeons to treat patients carrying HBV, HCV or HIV. The prime goal of preventive management must therefore be prevention of viral transmission from patients to health care providers. Better technique for invasive procedures, better gloves or other utilities and avoidance of unnecessary invasive investigations can decrease the risk. However, most important in the prevention of HBV is the preventive vaccination of health care providers. About $95 \%$ of all $\mathrm{HBV}$-infections of health care providers can be prevented by vaccination.

\section{References}

[1] R. Harpaz, L. Von Seidlein, F.M. Averhoff et al., Transmission of hepatitis B virus to multiple patients from a surgeon without evidence of inadequate infection control, N. Eng. J. Med. 334 (1996), 549-554.

[2] J.I. Esteban, J. Gómez, M. Martell et al., Transmission of hepatitis C virus by a cardiac surgeon, N. Eng. J. Med. 334 (1996), 555-560.

[3] J.L. Gerberding, The infected health care provider, N. Eng. J. Med. 334 (1996), 594-595.

\section{Immunisation in primary care centres and the temperature of the refrigerator (2)}

In this journal I reported recently about storage problems of vaccines in ill-working refrigerators in primary care centres in Colorado, USA [1,3]. General practitioner Andrew Jeremijenko reported 
similar problems with vaccine storage in the practices of general practitioners in Western Australia [2]. In a random sample of 50 general practices, $36 \%$ of the refrigerators used for vaccine storage recorded unacceptable temperatures. Most of these ill-working refrigerators had excessively low temperatures, damaging the vaccines by freezing. Jeremijenko also found a poor level of knowledge among staff members responsible for the vaccine storage. Commonly they did not pay attention to the temperature in the refrigerators.

This publication is another signal that more attention has to be given to good vaccine storage: better education and better monitoring of the refrigerator's temperature by digital maximum-minimum thermometers are required.

\section{References}

[1] E. Woodyard, L. Woodyard and W.A. Alto, Vaccine storage in the physician's office: a community study, J. Am. Board Fam. Pract. 8 (1995), 91-94.

[2] A. Jeremijenko, H. Kelly, B. Sibthorpe et al., Improving vaccine storage in general practice refrigerators, $B r . M e d . J$. 312 (1996), 1651-1652.

[3] A.F. Tempelaar, Immunisation in primary care centres: don't forget the freezer's temperature, in: Safety and risk in practice, Int. J. Risk \& Safety Med. 8 (1996), 185.

\section{ADRs in the perspective of time}

The risk of getting adverse drug reactions depends on several factors. There is an intrinsic risk related to the pharmacological properties of a drug. Extrinsic factors are the state of health and physiological factors in the drug-user, interaction with other drugs and food elements and the knowledge and the skills of the prescriber.

But, as Bert Leufkens and Ron Herings (Pharmacy Department of the University of Utrecht, The Netherlands) state, the risk of getting ADRs is also a function of time [1]. Newly registered drugs are more often given to patients with complicated disease histories and an increased risk-profile. The signalling of a serious adverse reaction gives an alert to the prescribers and decreases the risk by changing their prescribing behaviour. Long-term users have a lower risk than patients who use a drug for the first time, because vulnerable patients with high risk profiles are selected out in the group of long-term users (depletion of susceptibles). Estimating risk profiles of drugs, one must take into account this selection process of prescription ("confounding by indication").

\section{References}

[1] H.G. Leufkens and R.M.C. Herings, Vergelijking van veiligheidsprofielen van geneesmiddelen (Comparing safety profiles of drugs.), Pharm. Weekbl. 130 (1995), 800-801.

\section{Wooden tongue depressors caused fatal contamination with fungus}

Mitchell et al. reported an outbreak of an infection with Rhizopus microsporus in a neonatal unit of Birmingham Women's Hospital (UK), killing two premature babies and gravely injuring a third one (partial amputation of an arm). A fourth premature baby survived the infection but died afterwards 
from another complication [1]. The fungus was traced to wooden tongue depressors that were used as arm splints for securing intravenous lines. In an open box on a trolley at the unit, $10 \%$ of the tongue depressors carried Rhizopus microsporus, a fungus commonly found in old bread and fruit.

In another hospital in London the same fungus was found in 21 cultures after routine screening from children [2]. In the screening wooden tongue depressors were used. None of the children became ill. Also in this hospital the fungus was found on tongue depressors from open packs. It is unclear how the tongue depressors in the two hospitals became contaminated.

\section{References}

[1] S.J. Mitchell, J. Gray, M.E.I. Morgan et al., Nosocomial infection with Rhizopus microsporus in preterm infants: association with wooden tongue depressors, Lancet 348 (1996), 441-443.

[2] A. Tonks, Fatal outbreak traced to wooden tongue depressors, Br. Med. J. 312 (1996), 1186.

\section{Insufficient communication between general practitioners and medical specialists generates unnecessary diagnostic tests and referrals}

The communication between primary care practitioners and hospital specialists is the weakest chain in the referral system as used in several European countries. When the exchange of information is poor, patients are more at risk of undergoing unnecessary (or double) diagnostic tests or even of being referred unnecessarily again. In most countries medical education pays too little attention to this subject. Communication between primary care practitioners and hospital residents remains underdeveloped.

Arto Vehviläinen et al. from the University of Kuopio reported insufficient feedback communication from specialists to GPs in a rural district in Finland [1]. In their study among 1020 primary care practitioners ( $83 \%$ response) a referral rate was found of $5.8 \%$ in 58,760 patient-doctor contacts. The GPs did not receive feedback information in $42 \%$ of the referred cases. Surgical specialists did not send back a report in 53\% of the referrals. The communication between specialists and GPs was better in the rural districts than in the cities.

However, the insufficient communication was not only to be blamed on the medical specialists and hospitals. GPs with low referral rates, who paid much attention to their referral letters, more often received feedback information than GPs with high referral rates and referral letters that did not meet the standards.

Good communication between physicians has to do with a professional attitude. Although in most other European countries better agreements exist between primary care doctors and hospital residents, Hans Grundmeijer from the Dutch Society of GPs called it a structural problem [2]. A recently published study about feedback information from Dutch psychiatrists to GPs showed that more than $50 \%$ of the feedback letters which they wrote to GPs were incomplete [3].

Grundmeijer has called the relation between GPs and medical specialists an ambivalent one. They need each other, but there is also a competitive aspect. Many GPs have professional hospital experience whereas specialists have seldom worked in a general practice. Often specialists have no understanding of general practice. The input of patients in general practice is quite different from the input of patients to specialists. Medical specialists tend to have a pathophysiologic approach to the patient's problems where GPs have an epidemiological approach. GPs see a low prevalence of 'specialistic' diseases and 
they must play the gatekeeper role. GPs with a more pathophysiological approach more often refer patients unnecessary.

Understanding each other's working methods and good communication will improve the quality of care. Working together in a complementary model can prevent $10-20 \%$ of needless referrals!

\section{References}

[1] A.T. Vehviläinen, E.A. Kumpusalo and J.K. Takala, Feedback information from specialists to general practitioners in Finland, Eur. J. Gen. Pract. 2 (1996), 55-57.

[2] H. Grundmeijer, General practitioner and specialist: why do they communicate so badly?, Eur. J. Gen. Pract. 2 (1996), 53-54.

[3] E.W. Beyaert, M.M. Ubbink and A.J.A. Kaasenbrood, Matige kwaliteit van ontslagbrieven over psychiatrische patiënten (Mediocre quality of discharge letters concerning psychiatric patients), Ned. Tijdschr. Geneeskd. 140 (1996), 365-368. 